\title{
Medium- to Long-Term Impact of Rotavirus Vaccination on Hospital Care in Belgium: A 7-Year Follow-Up of the Rotavirus Belgium Impact Study (RotaBIS)
}

Baudouin Standaert · Danielle Strens · Ali Alwan · Marc Raes

To view enhanced content go to www.infectiousdiseases-open.com Received: October 20, 2015 / Published online: December 31, 2015

(C) GSK group of companies, Danielle Strens, Ali Alwan, Marc Raes 2015. This article is published with open access at Springerlink.com

\section{ABSTRACT}

Introduction: Rotavirus (RV) vaccination was introduced in Belgium in 2006. With the high uptake it had $(>85 \%)$, a sharp decline in hospitalizations was observed during the first years after vaccine introduction. The objective of this study was to investigate whether this

Electronic supplementary material The online version of this article (doi:10.1007/s40121-015-0099-1) contains supplementary material, which is available to authorized users.

B. Standaert $(\square)$

Health Economics Department, GSK Vaccines, Wavre, Belgium

e-mail: baudouin.a.standaert@gsk.com

B. Standaert

Unit of Pharmacoepidemiology and

Pharmacoeconomics (PE2), Department of

Pharmacy, University of Groningen, Groningen,

The Netherlands

D. Strens

Realidad bvba, Grimbergen, Belgium

A. Alwan

Biostatistics and Epidemiology Department,

Medstat, Ekeren, Belgium

M. Raes

Department of Pediatrics, Jessa Hospital, Hasselt, Belgium decline was maintained and to simulate projections.

Methods: The Rotavirus Belgium Impact Study allowed an analysis of the RV vaccine impact amongst children in 11 hospitals in Belgium over a 9-year period (2005-2013) with 2 years pre- and 7 years post-vaccine introduction. Results were compared by year and by subsequent birth cohort aging up to 5 years. The two different analysis methods helped dismantling the different (direct and indirect) effects of vaccine protection to simulate future hospitalization trends.

Results: During the whole observation period, $40,552 \mathrm{RV}$ detection tests were performed of which 5832 were positive (14.4\%). After RV vaccine introduction, a significant reduction in number of tests performed (-38\%) was combined with a dramatic drop in numbers of positive tests $(-76.6 \%)$. The decreases were spectacular during the first two years of vaccine introduction; after that period, the decrease flattened. Cross-sectional comparison with cohort data showed that the initial drop was heavily influenced by the herd effect of the vaccine. Cohort analysis demonstrated a low rate of residual disease over time, suggesting 
another infection source other than the child population.

Conclusion: The residual disease will be maintained in the community when a same vaccination strategy is continued over time, starting vaccination of children only at 6 weeks' time.

Funding: GlaxoSmithKline Biologicals SA.

Trial registration: ClinicalTrials.gov identifier, NCT01563146.

Keywords: Children;

Gastroenteritis;

Hospitalization; Rotavirus; Vaccination

\section{INTRODUCTION}

Rotavirus (RV) infection is one of the most common causes of diarrhea in young children less than 5 years old across the world, before the vaccine introduction in $2006[1,2]$. The infection is normally endemic but shows a higher frequency among young children during the winter period, especially in the more temperate climates of the developed world [3-5]. Severe consequences of RV gastroenteritis (RVGE) are more often observed in children under the age of 2 years, after which a dramatic drop in the number of diarrhea events is noticed [6]. After 5 years of age, children have normally acquired a natural immunity so that RV diarrhea is seldom reported [7-9].

RVGE is very contagious and its spread therefore remains difficult to control, even with rigorous application of primary hygienic measures [10]. As a result, the RVGE peaks are well-known as annually recurring public health problems. In Belgium for instance, before the introduction of the RV vaccine, the disease was causing a burden of around 70,000-75,000 diarrhea events per year (community-acquired and hospitalizations) in children under the age of 7 years $[11,12]$. The RV infection has some interesting features that make the contagion quite unique. Indeed, RV immunity is built up by successive infection exposures of which the first one is the most severe leading to acute symptoms but the following ones are progressively less severe $[9,13]$.

To reduce this public health burden, a radical change in disease prevention should be considered such as the early stimulation of infants' immunity between the ages of 6 and 10 weeks, thus providing protection before a first infection after which repeated exposures may assess a natural boost to immunity. This new prevention strategy can use RV vaccines available in Europe: a two-dose vaccine available since 2006, Rotarix ${ }^{\mathrm{TM}}$ (GSK), and a three-dose vaccine available since 2007, Rotateq (Merck and Co. Inc.). The first dose of the vaccine can be administered from 6 weeks of age, with a minimum interval of 4 weeks between subsequent doses [14, 15]. Although vaccination is recommended by European guidelines [16] and by the World Health Organization [17, 18], only 4 countries in Western Europe introduced the RV vaccine into their routine immunization schedules soon after the vaccine became available: Austria, Belgium, Finland, and Luxemburg [19]. By February 2014, national universal RV vaccination recommendations had been implemented in a few additional countries, including Estonia, Germany, Norway, and the United Kingdom (UK) [20].

Studies from Austria [21, 22], Finland [20, 23], and Belgium [24-26] have reported quite impressive reductions in hospitalizations 2-3 years after vaccine introduction combined with a vaccine herd effect. The medium- to long-term effect of the vaccine within the same at-risk group has not often been reported [27, 
28]. In the current study, we report about results of Belgium where the vaccine uptake was very high from start ( $>85 \%$ in the first year) and where cohorts of children $\leq 5$ years of age were followed from 2005 through 2013 (2 years pre-vaccination and 7 years post-vaccination) [29]. Such a long follow-up period should help in better understanding how the vaccine is working in real-life conditions. Hypothesizing that there is only one infection source (the children themselves) and that the vaccine effectiveness remains the same together with a well-maintained high vaccine uptake (>85\%), the RVGE hospitalization rate should decrease year after year, potentially leading to an elimination of the very severe disease cases very soon as disease modelers were speculating [30]. The present study aims to verify this hypothesis by analyzing and comparing the annual number of hospitalizations observed versus the expected ones through an appropriate modeling exercise in several birth cohorts over time. If major deviations between model-expected and observed results exist, the shape of the curve could help identifying a likely reason for the difference. Sources of curve deviation could include another source of infection not affected by the vaccine, vaccine waning, variable vaccine coverage rate, selection bias among some of the participating centers, or a combination of the different reasons.

\section{METHODS}

\section{Data Source}

Retrospective hospital database analyses were conducted (ClinicalTrials.gov identifier, NCT01563146) over an observation period of 9 years (2005-2013), at the same 11 hospitals in Belgium: 9 were general hospitals with a pediatric ward; 2 were pediatric-only hospitals. Among the 11 centers, 4 were university hospitals. The centers were distributed across the country and covered the three regions of Brussels, Flanders, and Wallonia. All of the centers combined had around 500 pediatric beds, representing $17 \%$ of the total of 2750 pediatric beds in Belgium.

Each participating center provided information on the laboratory assays used to detect RV. In each center, the following information were collected: Center code; children's date of birth; children's age; gender; date of sampling; RV tests (negative, positive); outcome (ambulatory or hospitalized); date of hospital admission and discharge; and length of hospital stay in days. The data were anonymized before any analysis occurred. All procedures followed were in accordance with the ethical standards of the responsible committee in each center. The approval for the study was obtained from each center and for each year the data were collected.

All children under 6 years of age who had an $\mathrm{RV}$ detection test performed at one of the participating centers from January 1, 2005 to May 31, 2013 were eligible for inclusion in the current analysis. The pre-vaccination study period was defined as from January 1, 2005 to December 31, 2006. The period from January 1, 2007 to May 31, 2013 was considered as the post-vaccination period (reimbursement of the vaccine was introduced in November 2006). The number of tests performed and the proportion of RV-positive tests were calculated for each participating center, per month, per year and for 7 different age-groups $(\leq 2$ months, $>2-12$ months, $>12$ months -2 years, $>2-3$ years, >3-4 years, $>4-5$ years, $>5$ years). Children aged $<12$ months were subdivided into two groups $(\leq 2$ months and $>2-12$ months) because the former group was 
too young for vaccination but could experience a herd protection effect once the vaccine is introduced.

\section{Data Analysis}

The data were analyzed in two different ways:

- Cross-sectional analysis The number of hospitalizations during the yearly peak periods (January until the end of May) for the 7 age-groups during the period 2005-2013 was summed up and reported annually. The data were compared by age-group and overall per year: pre-vaccination versus post-vaccination (1st year, 2nd year, $n$th year, ..., 7 th year postvaccination).

- Birth cohort analysis The number of hospitalizations during the peak period was noted in the birth cohort for the 1st and for each subsequent year of that cohort until the children were getting 5 years old. The results were summed up by year for each birth cohort and for the total follow-up period. The results were compared by subsequent birth cohorts. Using this approach, we could report 3 vaccinated subsequent birth cohorts getting 5 years old and 5 vaccinated subsequent birth cohorts getting 3 years old. We also compared those results with the pre-vaccination period but cross-sectional only as those data were not under the influence of RV vaccination.

Two important assumptions underlying the comparison of the annual number of $\mathrm{RV}$-positive tests were that the catchment area for each of the participating centers remained the same across the whole observation period of 9 years and that no change in disease management behavior for testing the children $\leq 5$ years old on RV disease occurred during that period. It means that if fewer tests were performed once the vaccine has been introduced, this has mainly to do with less suspected cases presenting themselves to the hospital unit and not with a change in behavior of the physician who was less likely to perform $\mathrm{RV}$ tests once the vaccine was introduced. Therefore, the most relevant value to compare between the years is the accumulated number of $\mathrm{RV}$-positive tests and not the proportion of RV-positive tests.

\section{Model Simulation}

To well-understand the real impact the vaccine has, this can best be achieved by comparing observed results with a model simulation in which we separately control the different aspects that could impact on the outcome (hospital reduction) such as changing the vaccine efficacy (VE) over time, initiating a second source of infection not being affected by the vaccine, changing the vaccine waning rate, or changing the vaccine uptake per year. For doing that comparison we selected from the observed data the birth cohort follow-up data up to the age of 3 years to obtain enough data-points over time.

A time difference equation model was developed based on the initial data collection of the first years of observation. An analysis was then simulated in which the decrease in hospital numbers for the first few years fitted the observed data with fixed parameters over time, a calibration process:

$$
x_{t+1}=\eta x_{0}+(1-\eta)\left(1-\rho \sigma_{t} \omega^{t}\right) x_{t}
$$

in which: $x_{t}$ is the number of hospitalizations in year $t ; x_{r}$ is the residual number of hospitalizations such that $x_{r}=\eta x_{0}$ where $\eta$ is the fraction of hospitalizations caused by another source of infection; $\rho$ is a fixed 
parameter equivalent to the VE; $\sigma_{t}$ is the vaccine coverage rate in year $t ; \omega$ is a fixed parameter reflecting VE waning over time; and $t$ is the time indicator expressed in year units.

With this model, we may easily adjust the shape of the simulated curve changing separately $\rho$ or the VE, the vaccine uptake $\left(\sigma_{t}\right)$ at specific $t$ time points, the vaccine waning by decreasing $\omega$, and the residual disease caused by another source of infection by changing $\eta$. The simulated shape can then be compared with the observed data and the best fit is selected for the most plausible scenario of projected future hospitalizations related to the disease. No statistically significant difference between the curves were tested using Mann-Whitney $U$ testing $(P<0.05)$ and by calculating $R^{2}$ value (Stata 11.2; StataCorp LP, College Station, TX, USA).

\section{Outcomes}

An annual comparison of cross-sectional data will identify the importance of the herd effect generated by the vaccine. Analyzing and comparing successively vaccinated birth cohorts over time will indicate whether the vaccine wanes. Comparison of the summary measures of vaccinated birth cohorts per year with simulated predictions will test the hypothesis about different sources of infection. Finally, the proportional difference in $\mathrm{RV}$-positive tests between pre- versus the most recent post-vaccination period analyzed (2012-2013) across the 11 participating centers will identify any selection bias in participating centers that may explain why residual disease could be observed over time. Results are tested for statistical significance using Chi-square tests (using Stata 11.2) for trend of proportional data, with a statistical significance level of $P<0.05$ [31].

\section{RESULTS}

\section{Cross-Sectional Analysis}

Among the 11 participating centers, 40,552 $\mathrm{RV}$ tests were conducted over the observation period of 9 years, with a much higher frequency during the peak months. The overall number of RV-positive tests recorded during that period was 5832 (14.4\%). Over the years, the overall number of tests performed significantly decreased from 6278 tests in 2006 during the pre-vaccination period to 3893 tests in 2012 during the post-vaccination period, representing a reduction of $38 \%$. Moreover, the absolute number of RV-positive tests decreased from 1399 tests in 2006 during the pre-vaccination period to 327 tests in 2012 during the post-vaccination period, representing a reduction of $76.6 \%$. The decrease in RV-positive tests was extremely sharp during the first 2-3 years after the introduction of the vaccine and then flattened over time, compared with the more linear decrease in the number of RV-negative tests over time (Fig. 1).

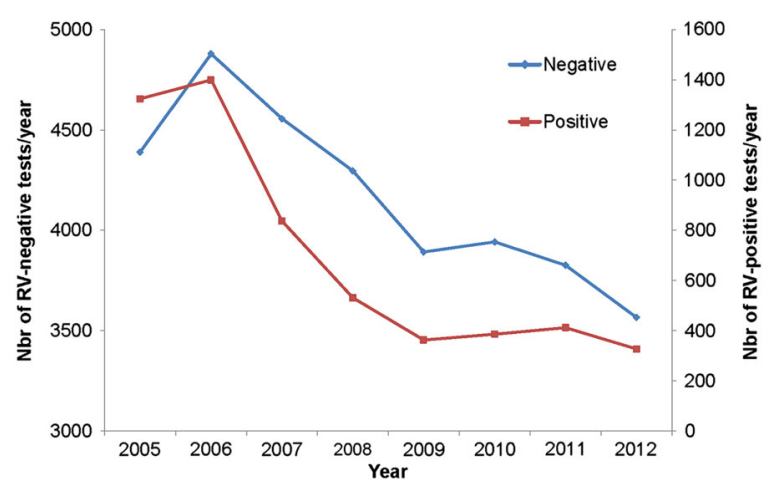

Fig. 1 Number of RV-negative and RV-positive tests performed per year in the 11 participating centers $\mathrm{Nbr}$ number, $R V$ rotavirus 
The non-linear reduction in the number of RV-positive tests over time was statistically significant $\left(\chi^{2}=215.95 ; P<0.0001\right)$.

The number of RV-positive tests is shown by age-group and year in Fig. 2. There was a sharp drop in the number of RV-positive cases in the first year after the introduction of RV vaccination, especially in the vaccinated age-groups ( $>2-12$ months and $>12$ months -2 years) which represent nearly $78 \%$ of the entire study population. There was also a drop in the number of positive cases in children who were either too young ( $\leq 2$ months) or too old ( $>6$ months) to be immunized.

\section{Birth Cohort Analysis}

Table 1 shows the numbers of RV-positive tests by age-group and by year for each peak period (January until end of May). The accumulated data represent $85 \%$ of the total $\mathrm{RV}$-positive tests, with the remaining $15 \%$ observed in the other months of the year, especially November and December.

In 2007, the cross-sectional results indicated a total of $710 \mathrm{RV}$-positive tests, which was $44 \%$ lower than the year before when no vaccine was provided (there were $1271 \mathrm{RV}$-positive tests in 2006). By following the birth cohort

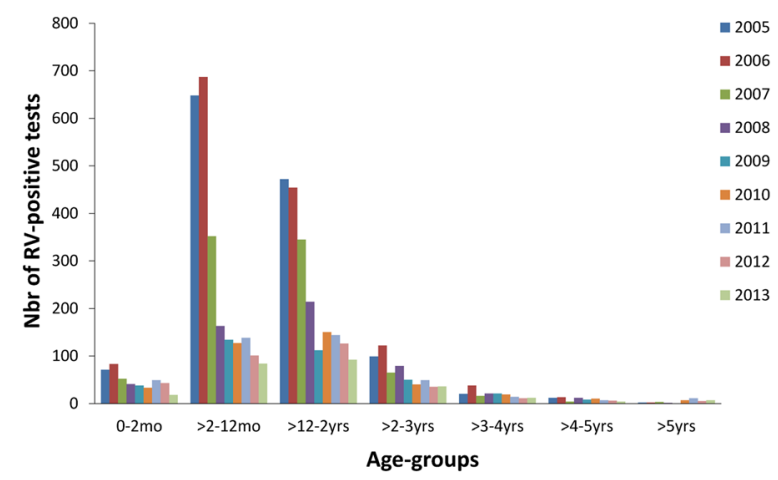

Fig. 2 Distribution of RV-positive tests by age-group and year. $N b r$ number, $R V$ rotavirus, mo months, yrs years from 2007 to 2012, and summing the values year-by-year with increasing age in the Table $(69+305+199+44+16+5+2), \quad 640$ $\mathrm{RV}$-positive tests are reached. As expected, this is lower than the cross-sectional result of 2007. There is a difference between the two values (710 versus 640) caused by the herd effect only (marked as yellow cells in the table for the cross-sectional calculation) versus the age-groups subject to direct vaccine effect plus the herd effect (marked as light brown cohort cells; Table 1).

A herd effect was maintained after the first 5 years of the vaccination program, as shown by the continued reduction in RV-positive tests seen in children $<2$ months of age who are too young for vaccination (first row, yellow cells; Table 1).

A particularly interesting finding in the cohort analysis is that the data do not show any significant additional drop in the early age-groups ( $>2-12$ months and 12 months- 2 years) after the large decrease of the first 2 years. This indicates that the rate of decrease in hospitalization changes over time, which indirectly reveals that another factor must influence the process of RV infection in this child population.

\section{Model Simulations}

Figure 3 compares first-year observed results (Fig. 3a) with simulations of having another source of infection in the population (Fig. 3b), having no other source of infection in the population (Fig. 3c), vaccine waning (Fig. 3d), and having a different vaccine uptake scenario (Fig. 3e).

There is a good fit between the observed data and the simulations for the first 3 years when the $\rho$-factor in the simulation equation equals 0.5 and no other source of infection was present 
Table 1 CS and by BC analysis of the rotavirus-positive tests by age and year during the peak period (January to end of April)

\begin{tabular}{|c|c|c|c|c|c|c|c|c|c|c|}
\hline \multirow[b]{2}{*}{ Year/Age } & \multicolumn{2}{|c|}{$\begin{array}{c}\text { Unvaccinated } \\
\text { years }\end{array}$} & \multicolumn{7}{|c|}{ Vaccinated years } & \multirow[b]{2}{*}{ Total } \\
\hline & 2005 & 2006 & 2007 & 2008 & 2009 & 2010 & 2011 & 2012 & 2013 & \\
\hline$\leq 2$ months & 84 & 100 & 69 & 45 & 41 & 32 & 44 & 31 & 28 & 474 \\
\hline$>2-12$ months & 551 & 634 & 305 & 127 & 108 & 101 & 95 & 80 & 82 & 2083 \\
\hline$>12$ months -2 years & 367 & 381 & 266 & 199 & 88 & 116 & 107 & 88 & 89 & 1701 \\
\hline$>2-3$ years & 82 & 111 & 51 & 59 & 44 & 23 & 38 & 29 & 31 & 468 \\
\hline$>3-4$ years & 16 & 34 & 14 & 18 & 16 & 16 & 7 & 9 & 13 & 143 \\
\hline$>4-5$ years & 9 & 10 & 3 & 12 & 7 & 7 & 5 & 5 & 3 & 61 \\
\hline$>5$ years & 2 & 1 & 2 & 1 & 0 & 6 & 10 & 2 & 7 & 31 \\
\hline Total CS & 1111 & 1271 & 710 & 461 & 304 & 301 & 306 & 244 & 253 & 4961 \\
\hline Total BC & & & & & & & & 640 & 302 & \\
\hline
\end{tabular}

Brown cells: vaccine effect evaluated by cohort; yellow cells: herd protection only; white cells: no vaccine effect. Colored bold numbers (red and dark brown) indicate how the BC totals are calculated per follow-up until the BC reaches 5 years. Only two BCs (2007 and 2008) have reached the full 5 year follow-up period under the vaccine effect. Comparing the full-vaccine effect has a bigger impact by BC than CS by year up to the age of 5 years (710 CS versus $640 \mathrm{BC}$ 1st evaluation and $461 \mathrm{CS}$ versus $302 \mathrm{BC} 2$ nd evaluation)

$B C$ birth cohort, $C S$ cross-sectional

(Fig. 3c). Under such scenario we could normally foresee an elimination of the disease over a few years as it was hypothesized by some modelers. However, a much better overall good fit of the whole observed curve shape (Fig. 3b) was obtained if the additional source of infection with residual disease ( $\eta$-factor) was introduced in addition to the $\rho$-factor. The $\eta$-factor was estimated at $12 \%$ of the hospitalizations in the equation $\left(R^{2}=0.989 ; \quad\right.$ Mann-Whitney $U$ rank-sum test between observed and simulated curve, $Z$ stat $=0.368, P=0.713)$.

For vaccine waning (Fig. 3d), we first simulated a decrease of $10 \%$ of $\omega$ per year. To obtain a perfect fit with the observed data the annual waning would have to reach $35 \%$, starting in the second year of vaccination.

Varying the vaccine uptake from $85 \%$ to $65 \%$ in the 3rd year doesn't affect the curve (Fig. 3e) so much as the biggest drop in hospitalization occurs in the first years and what happens thereafter appears having a marginal effect. The point to make here is that any decrease in vaccine uptake later on, cannot explain the observed curve as it is now.
Finally the proportion of RV-positive tests across all the participating hospital centers was higher during the pre-vaccination period (2005-2006) than during the post-vaccination period (2012-2013) as expected. The average difference between 2005-2006 and each of 2012 and 2013 was 0.163 (minimum 0.10 to maximum 0.24 ) and 0.178 (minimum 0.07 to maximum 0.30), respectively. No center was noticeably an outlier (see Fig. 4).

\section{DISCUSSION}

This analysis of medium- to long-term impact of $\mathrm{RV}$ vaccination on specific tests measured annually in the same 11 hospital centers in Belgium has identified several interesting features.

First, there is a large reduction in frequency of RV disease during the normal seasonal peak period after vaccination of the first birth cohort had started. The decrease of $70-80 \%$ in $\mathrm{RV}$-positive tests, compared with the period of no vaccination, is achieved within 2 years after vaccine introduction. These findings have 


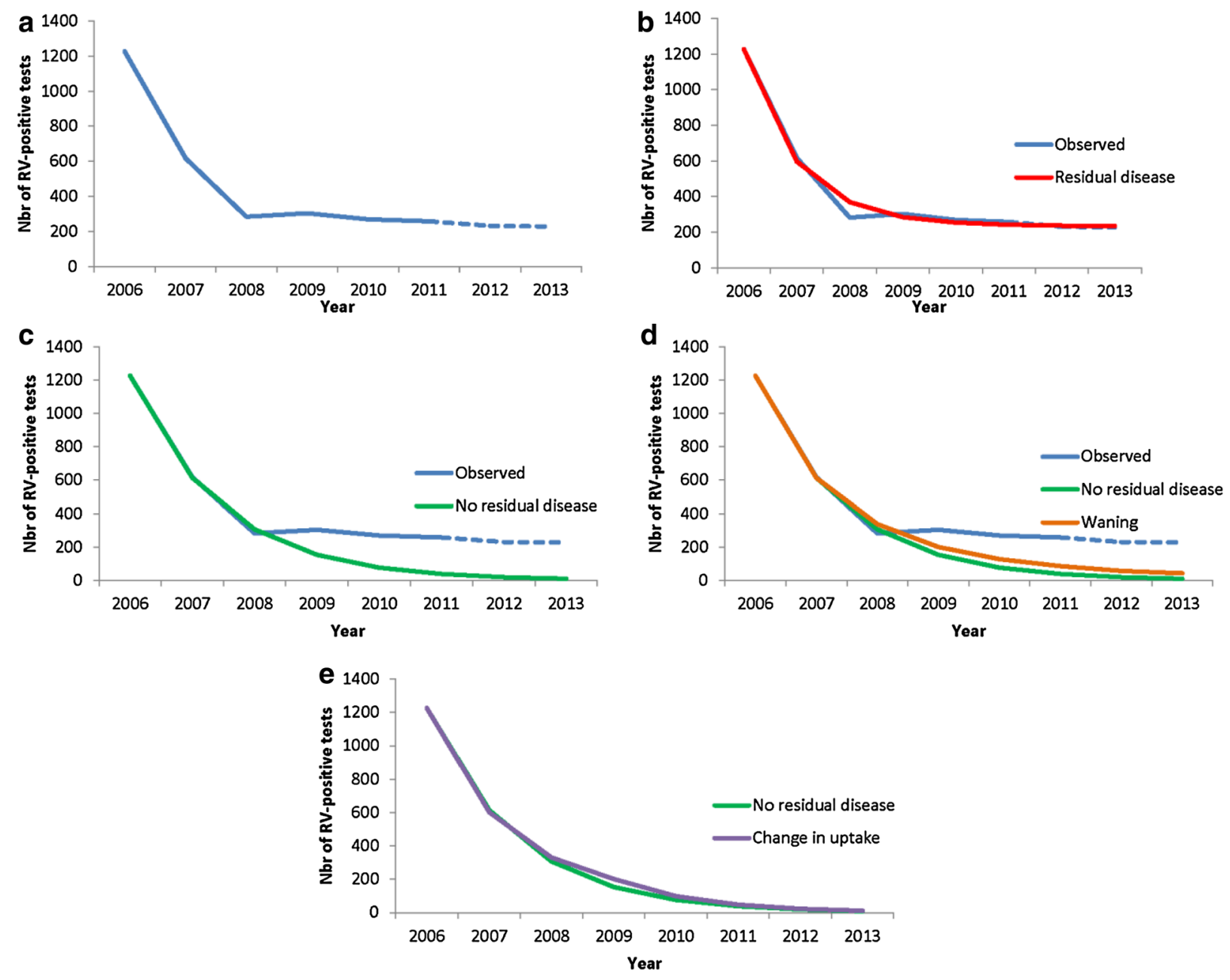

Fig. 3 Comparing observed data (a) with simulations of adding residual disease over time (b); with fixed reduction in RV-positive tests without residual disease (c); vaccine

waning $(-10 \%$ per year $)(\mathbf{d})$; changing vaccine coverage rate $(85 \%$ to $65 \%)$ (e). $N b r$ number, $R V$ rotavirus

already been reported in previous publications for Belgium [25, 32]. After that large initial drop however, subsequent annual decreases are more modest (around 10-15\% per age-group). A similar early vaccine effect (the sharp drop in the first year) has been observed in the UK during the first year after the vaccine introduction [33]. The decline is more spectacular during the first year than in the present study. This could be due to the start date of the vaccination campaign in the UK, which was planned by the end of the second quarter the year before the start of the next peak season. In Belgium, vaccination began much closer to the next peak season, namely in the fourth quarter of the year [34].

Second, in addition to the important direct vaccine effect seen in the first vaccinated birth cohort, we also observe, during the same period, a substantial drop in the unvaccinated age-groups (i.e., children too young or too old to be vaccinated, as shown in Table 1). This phenomenon clearly indicates the high transmission rate of the virus between the 


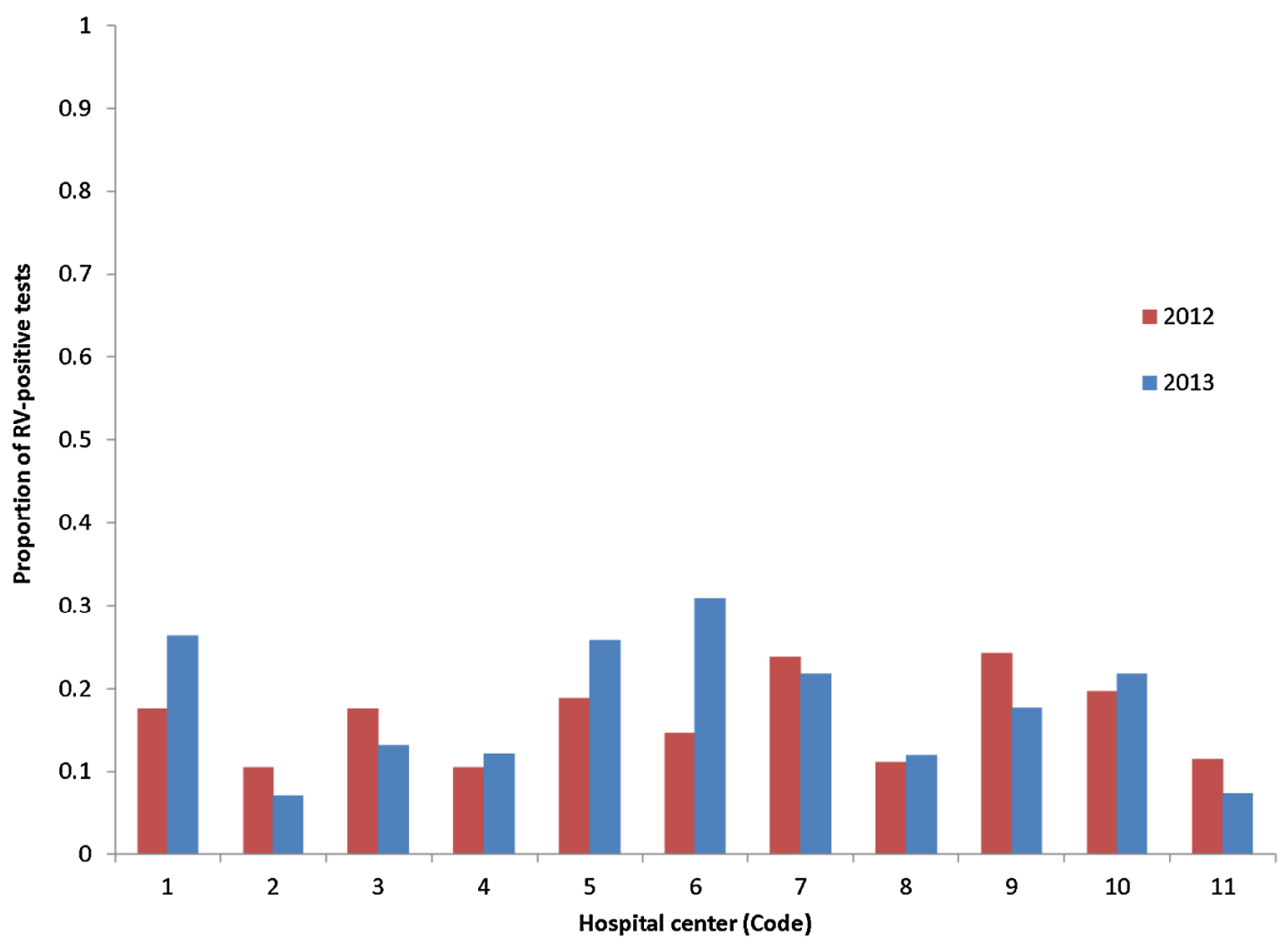

Fig. 4 Difference in proportion of RV-positive tests during the post-vaccination period (2012 and 2013) compared with the pre-vaccination period (2006) in each hospital center (code). $R V$ rotavirus

different age-groups, resulting in a high indirect herd protection effect of the vaccine during the first years of the vaccination program until the whole at-risk group (aged up to 5 years old) is covered. The herd protection during the first and second year after the vaccine introduction is estimated at $40 \%(233 / 561)$ and $15 \%(123 /$ $810)$, respectively, relative to the overall reduction in hospital events per year as compared to the baseline values of 2006 .

The overall drop in disease events was spectacular during the first two years, because the younger age-groups targeted by the vaccine program are the groups most affected by $\mathrm{RV}$ disease (peak incidence rates) and are the highest receivers and transmitters of the virus to other age-groups. Virus transmission within these age-groups and to other age-groups was directly and indirectly reduced by the vaccine.
Once the at-risk group has been vaccinated, herd protection in the older age-groups would be expected to disappear, as children in this group would have been vaccinated when they were younger. This would leave the herd effect being present only in children $\leq 2$ months of age (who are too young for vaccination) as an additional benefit sustained over time.

With 7 years of real-world observations after vaccine introduction, this study provides information about the likely source of infection of RV disease in the child population. The results split by age-group in Fig. 2 indicate that the role of the vaccine is primarily to stop the spread of the infection within the child population. The vaccine fulfills that task very well, as it induces a high level of herd protection across the different unvaccinated age-groups during the same period. To obtain such a high 
vaccine impact, the main source of infection must be within the children themselves as it blocks the root cause of infection transmission. However, the amount of indirect effect depends heavily on how children are normally nurtured during that period. For example, do they attend day-care centers and at what starting age, do they have regular contact with other children elsewhere, and are different disease patterns observed between different age-groups if child management or behavior changes? These questions affect the likely sources of infection and patterns of disease transmission between and within age-groups, and would be valuable areas for further research. Finally, the different observed rates of disease reduction in subsequent years across different age-groups are a signal that different infection forces operate within the child population. The most plausible explanation, simulated in Fig. 3, is that there are additional sources of infection that are seen once most of the herd effect has faded away after the RV-vaccine program has been in place for a few years and all the at-risk children have been vaccinated. This scenario appears much more likely than a vaccine waning scenario, because an annual decrease of $35 \%$ in vaccine effect starting 1 year after its introduction must occur to fit the observed data. Another possibility could be that the vaccination coverage rate fluctuated over time, but it is unlikely that that potential disturbance may impact so heavily the outcome results. In addition, the vaccine coverage rate in Belgium remained stable and quite high during the whole observation period (>86\%, [32]).

These indications of additional sources of infection suggest that the disease and the virus will not be easily eliminated unless the other sources of infection can be targeted by different vaccination strategies.
A cohort analysis illustrates effects within the child population over time, including the dynamics of indirect vaccine impact. This type of investigation is more sensitive and better able to identify the real-world benefit of the vaccine than using VE data obtained through randomized clinical trials, where the control group may be influenced by the herd protection. This may reduce the measured VE, as seen in the European trial [35].

Following a first birth cohort over time should normally demonstrate a larger reduction of $\mathrm{RV}$-positive tests than a first-year cross-sectional evaluation, because the vaccinated birth cohort includes a mixture of direct and indirect vaccine effects in each subsequent year if the coverage rate is not $100 \%$. In contrast, the cross-sectional analysis only includes the measured herd effect in addition to the first-year direct effect of vaccination of a small age-group. Thus, we would expect to observe a larger effect in the birth cohort analysis than the cross-sectional analysis when comparing the two datasets, which is consistent with what is observed in this study. However, cross-sectional and cohort data would be expected to reach the same end result for the sum of the different at-risk age-groups as soon as all the children from all the different at-risk age-groups have been vaccinated, approximately 10 years from the start [36, 37]. Comparing birth cohort and cross-sectional analyses can also allow estimating the magnitude of the pure herd effect that can be generated by the vaccine in this disease.

Finally, all centers responded to the vaccine in a similar way over time. The observation of a residual disease burden could have been linked to specific centers that did not apply the same vaccination strategy in their catchment area, or 
to potential insourcing in some specific areas of unvaccinated children from outside Belgium where vaccination is not yet routinely performed. These possibilities were not measured in the present study.

A limitation of the current study is that we do not fully control the denominator of the study, and thus we assume that the target population has not significantly changed over the 9 years of the study period. For a small country like Belgium, with a stable population (the birth cohort may change by less than $0.6 \%$ per year), this assumption is reasonable. Another assumption is that no change behavior in testing the children for RV infection appeared over time after the introduction of the vaccine.

\section{CONCLUSIONS}

The analysis here presented may provide the evidence for another source of RV infection after the introduction of the RV-vaccine that exists outside the child population such as care-givers at home or day-care centers, even the animal environment. This source appears to be less spectacular in spreading the disease in the child population than the transmission within the age-group. It may also less likely be significantly influenced by vaccination because the current strategy may not directly touch these reservoirs.

The results of this study help to fill an information gap about the impact of $\mathrm{RV}$ vaccination over the medium- to long-term. The main features reported are the sustained reduction in hospitalization. The new finding about different sources of infection in the child population reveals that it may be difficult to reduce the disease to very low levels. A residual disease presence observed over time means that we need to continue to monitor the events each year to detect any new developments.

\section{ACKNOWLEDGMENTS}

GlaxoSmithKline Biologicals SA was the funding source and was involved in all stages of the study conduct and analysis (ClincialTrials.gov identifier, NCT01563146). GlaxoSmithKline Biologicals SA also took in charge all costs associated with the development and the publishing of the present manuscript, including the article processing charges. All authors had full access to the data and the corresponding author had final responsibility to submit for publication. All named authors meet the International Committee of Medical Journal Editors (ICMJE) criteria for authorship for this manuscript, take responsibility for the integrity of the work as a whole, and have given final approval to the version to be published. The authors would like to thank the investigators of The Rotavirus Belgium Impact Study (RotaBIS study) group who participated in this study: Marc Verghote, Centre Hospitalier Régional de Namur, Namur; Eddy Bodart, Cliniques Universitaires, Université catholique de Louvain (UCL) de Mont Godinne, Yvoir; Stefaan Van Eldere, Imeldaziekenhuis, Bonheiden; Patricia Vandecandelaere, Regionaal Ziekenhuis Jan Yperman - O.L.Vrouw, Ieper; Peggy Bruynseels, Ziekenhuis Netwerk Antwerpen, Antwerpen; Jose Ramet, Universitair Ziekenhuis Antwerpen, Edegem; Hajer Lassoued, Clinique Notre Dame, Charleroi. The authors thank Gregory Collet and Sarah Fico (both Business \& Decision Life Sciences, Brussels, Belgium, on behalf of GSK Vaccines, Wavre, Belgium) for editorial assistance and manuscript 
coordination. The authors would like to also thank Thibaut Dort (Navigha, Brussels, Belgium, on behalf of GSK Vaccines, Wavre, Belgium) for his latest contribution of reviewing the statistical analysis program.

Disclosures. Baudouin Standaert is an employee of the GSK group of companies and holds stock in the GSK group of companies. Danielle Strens' institution received consulting fees from the GSK group of companies for work under consideration for publication and outside the submitted work. Ali Alwan's institution received consulting fees from the GSK group of companies for work under consideration for publication and outside the submitted work. Marc Raes' institution received a consulting fee or honorarium from the GSK group of companies for the submitted work. Outside the submitted work, Marc Raes received funding from the GSK group of companies for board membership and lectures and has received funding for travel, accommodation and meetings expenses.

Compliance with ethics guidelines. The data were anonymized before any analysis occurred. All procedures followed were in accordance with the ethical standards of the responsible committee in each center. The approval for the study was obtained from each center each year the data were collected.

Open Access. This article is distributed under the terms of the Creative Commons Attribution-NonCommercial 4.0 International License (http://creativecommons.org/licenses/ by-nc/4.0/), which permits any noncommercial use, distribution, and reproduction in any medium, provided you give appropriate credit to the original author(s) and the source, provide a link to the Creative Commons license, and indicate if changes were made.

\section{REFERENCES}

1. Karsten C, Baumgarte S, Friedrich AW, et al. Incidence and risk factors for community-acquired acute gastroenteritis in north-west Germany in 2004. Eur J Clin Microbiol Infect Dis. 2009;28(8):935-43.

2. Parashar UD, Gibson CJ, Bresse JS, Glass RI. Rotavirus and severe childhood diarrhea. Emerg Infect Dis. 2006;12(2):304-6.

3. Iturriza-Gomara $\mathrm{M}$, Dallman $\mathrm{T}$, Banyai $\mathrm{K}$, et al. Rotavirus surveillance in Europe, 2005-2008: web-enabled reporting and real-time analysis of genotyping and epidemiological data. J Infect Dis. 2009;200(Suppl 1):S215-21.

4. Forster J, Guarino A, Parez N, et al. Hospital-based surveillance to estimate the burden of rotavirus gastroenteritis among European children younger than 5 years of age. Pediatrics. 2009;123(3):e393-400.

5. Van Damme P, Giaquinto C, Huet F, et al. Multicenter prospective study of the burden of rotavirus acute gastroenteritis in Europe, 2004-2005: the REVEAL study. J Infect Dis. 2007;195(Suppl 1):S4-16.

6. Giaquinto C, Van Damme P, Huet F, et al. Clinical consequences of rotavirus acute gastroenteritis in Europe, 2004-2005: the REVEAL Study. J Infect Dis. 2007; 195(Supplement 1):S26-35.

7. Bresee JS, Glass RI, Ivanoff B, Gentsch JR. Current status and future priorities for rotavirus vaccine development, evaluation and implementation in developing countries. Vaccine. 1999;17(18): 2207-22.

8. Huilan S, Zhen LG, Mathan MM, et al. Etiology of acute diarrhoea among children in developing countries: a multicentre study in five countries. Bull World Health Organ. 1991;69(5):549-55.

9. Velazquez FR, Matson DO, Calva JJ, et al. Rotavirus infections in infants as protection against subsequent infections. $\mathrm{N}$ Engl J Med. 1996; 335(14):1022-8.

10. Diez-Domingo J, Martin IO, Sanz AB, et al. Rotavirus gastroenteritis among children under five years of age in Valencia, Spain. Pediatr Infect Dis J. 2006;25(5):455-7. 
11. Bilcke J, Beutels P, De Smet F et al. KCE reports 54B: Vaccination des nourrissons contre le rotavirus en Belgique-Analyse coût-efficacité. Bruxelles: Centre fédéral d'expertise des soins de santé (KCE); 2007. Report No.: KCE reports 54B, Dépot légal D2007/ $10.273 / 10$

12. Bilcke J, Van DP, Beutels P. Cost-effectiveness of rotavirus vaccination: exploring caregiver(s) and "no medical care" disease impact in Belgium. Med Decis Making. 2009;29(1):33-50.

13. Velazquez FR. Protective effects of natural rotavirus infection. Pediatr Infect Dis J. 2009;28(3 Suppl):S54-6.

14. Anderson EJ. Prevention and treatment of viral diarrhea in pediatrics. Expert Rev Anti Infect Ther. 2010;8(2):205-17.

15. Chandran A, Fitzwater S, Zhen A, Santosham M. Prevention of rotavirus gastroenteritis in infants and children: rotavirus vaccine safety, efficacy, and potential impact of vaccines. Biologics. 2010;4:213-29.

16. Vesikari T, Van Damme P, Giaquinto C, et al. European society for paediatric infectious diseases/ European society for paediatric gastroenterology, hepatology, and nutrition evidence-based recommendations for rotavirus vaccination in Europe: executive summary. J Pediatr Gastroenterol Nutr. 2008;46(5):615-8.

17. Rotavirus vaccines WHO position paper: January 2013-Recommendations. Vaccine. 2013;31(52):6170-1.

18. World Health Organization. Meeting of the immunization Strategic Advisory Group of Experts, April 2009-conclusions and recommendations. Wkly Epidemiol Rec. $2009 ; 23(84): 220-36$.

19. Van Damme P, Van der Wielen M, Ansaldi F, et al. Rotavirus vaccines: considerations for successful implementation in Europe. Lancet Infect Dis. 2006;6(12):805-12.

20. Parez N, Giaquinto C, Du RC, et al. Rotavirus vaccination in Europe: drivers and barriers. Lancet Infect Dis. 2014;14(5):416-25.

21. Paulke-Korinek M, Kundi M, Rendi-Wagner P, et al. Herd immunity after two years of the universal mass vaccination program against rotavirus gastroenteritis in Austria. Vaccine. 2011;29(15):2791-6.

22. Paulke-Korinek M, Kollaritsch H, Aberle SW, et al. Sustained low hospitalization rates after four years of rotavirus mass vaccination in Austria. Vaccine. 2013;31(24):2686-91.

23. Vesikari T, Sutherland D, Jackson AEM. Report of the 'European Expert Meeting on Rotavirus Vaccination', Tampere, Finland, 19-20 May 2009. Vaccine. 2009;27(52):7222-7.

24. Hanquet G, Ducoffre G, Vergison A, et al. Impact of rotavirus vaccination on laboratory confirmed cases in Belgium. Vaccine. 2011;29(29-30):4698-703.

25. Raes $M$, Strens D, Vergison A, Verghote $M$, Standaert B. Reduction in pediatric rotavirus-related hospitalizations after universal rotavirus vaccination in Belgium. Pediatr Infect Dis J. 2011;30(7):e120-5.

26. Zeller M, Rahman M, Heylen E, et al. Rotavirus incidence and genotype distribution before and after national rotavirus vaccine introduction in Belgium. Vaccine. 2010;28(47):7507-13.

27. Zlamy M, Kofler S, Orth D, et al. The impact of Rotavirus mass vaccination on hospitalization rates, nosocomial Rotavirus gastroenteritis and secondary blood stream infections. BMC Infect Dis. 2013;13:112.

28. Vesikari $\mathrm{T}$, Uhari $\mathrm{M}$, Renko $\mathrm{M}$, et al. Impact and effectiveness of RotaTeq $(\mathrm{R})$ vaccine based on 3 years of surveillance following introduction of a rotavirus immunization program in Finland. Pediatr Infect Dis J. 2013;32(12):1365-73.

29. Braeckman $\mathrm{T}$, Theeten $\mathrm{H}$, Lernout $\mathrm{T}$ et al. Rotavirus vaccination coverage and adherence to recommended age among infants in Flanders (Belgium) in 2012. Eurosurveillance. 2014;19(20) (pii=20806).

30. Atkins KE, Shim E, Pitzer VE, Galvani AP. Impact of rotavirus vaccination on epidemiological dynamics in England and Wales. Vaccine. 2012;30(3):552-64.

31. Fleiss J, Levin B, Paik M. Statistic methods for rates and proportions. Probabilties and statistics. Hoboken:New Jersay Wiley Series edn. Wikey Series; 2003.

32. Standaert B, Gomez JA, Raes M, et al. Impact of rotavirus vaccination on hospitalisations in belgium: comparing model predictions with observed data. PLoS One. 2013;8(1):e53864.

33. Owain J. Vaccine Knowledge Project. Available from: www.ovg.ox.ac.uk/rotavirus-vaccine. Accessed 23 Dec 2014

34. Public Health England. Summary of Surveillance of norovirus and rotavirus vaccination. Available 
from: http://www.hpa.org.uk/Topics/Infectious Diseases/InfectionsAZ/Rotavirus/Epidemiological Data/. Accessed 27 Nov 2014.

35. Vesikari T, Karvonen A, Prymula R, et al. Efficacy of human rotavirus vaccine against rotavirus gastroenteritis during the first 2 years of life in European infants: randomised, double-blind controlled study. Lancet. 2007;370(9601):1757-63.
36. Standaert B, Demarteau N, Talbird S, Mauskopf J. Modelling the effect of conjugate vaccines in pneumococcal disease: cohort or population models? Vaccine. 2010;28(Suppl 6):G30-8.

37. Ethgen O, Standaert B. Population- versus cohort-based modelling approaches. Pharmacoeconomics. 2012; 30(3):171-81. 